\title{
Fabrication and Evaluation of Silicon Nanowire Photodetectors on Flexible Substrate for Retinal Prosthetic System
}

\author{
Sangmin Lee**, Suk Won Jung*,, ${ }^{*}$ Sungil Park, Jaehyun Ahn, \\ Seok June Hong, Hyung Jung Yoo, Min Ho Lee' and Dong-il "Dan" Cho* \\ ISRC/ASRI, Department of Electrical and Computer Engineering, Seoul National University, \\ Gwanak-ro 1, Gwanak-gu, Seoul 151-744, Republic of Korea \\ ${ }^{1}$ Medical IT Convergence Research Center, Korea Electronics Technology Institute, \\ Yatap-dong, Bundang-gu, Seongnam 463-816, Republic of Korea
}

(Received 9 December 2011; accepted 13 February 2012)

Key words: silicon nanowire, photodetector, electrical stimulation, retinal prosthesis

Retinal degenerative diseases result in a progressive degeneration of photoreceptors in the retina and eventually lead to complete blindness. In this study, we develop a novel silicon nanowire photodetector on a flexible substrate to replace the function of photoreceptors on the retina and restore vision for the blind. An extremely high surfaceto-volume-ratio characteristic of the nanowire enables the development of highly sensitive photonic sensors. Therefore, a nanowire-based photodetector can be used as a stand-alone retinal stimulation system without an external camera. The fabricated silicon nanowire photodetector is measured to have an average photoresponsivity of $1 \times 10^{4} \mathrm{~A} / \mathrm{W}$ in a wide range of light intensities and wavelengths. Also, the optical characteristics of the silicon nanowire photodetector are well maintained on a flexible substrate, which is verified by comparing the photosensitivity before and after the substrate transfer from a rigid form to a flexible form. To evaluate the mechanical durability of the flexible silicon nanowire photodetector, mechanical bending tests are performed. The optical performance of the flexible photodetector is well preserved during bending for 200 times. The presented performance evaluation results show that the proposed nanowire-based photodetector can be used for the implementation of the retinal prosthetic system. The results of this proposed novel method can point the way to a revolutionary break-through in artificial retina research.

"Corresponding author: e-mail: dicho@snu.ac.kr

${ }^{* *}$ Joint first author 


\section{Introduction}

Retinal degenerative diseases such as age-related macular degeneration (ARMD) and retinitis pigmentosa (RP) result in a progressive degeneration of photoreceptors in the retina and eventually lead to complete blindness. ${ }^{(1,2)}$ A number of pharmaceutical treatments have been developed to prevent the progress of or cure the degenerative disease itself. However, the pharmaceutical treatment results to date have only shown the slowing down of the progress and no method to stop the progress has yet been established. ${ }^{(3)}$

Despite a near-total loss of the macular photoreceptors in the outer nuclear layer and even in the final stage of RP or ARMD, there are reports that the inner nuclear and retinal ganglion layers are partially preserved, retaining nearly 30 to $80 \%$ of the neurons found in a healthy eye. ${ }^{(4-6)}$ Also, these results support that the approach of restoring vision by electrical stimulation of the surviving neurons using neural prosthetic devices may be viable as a vision-recovery methodology in retinal degenerative diseases. ${ }^{(7,8)}$ The electrical stimulation typically involves a biphasic current waveform for the purpose of driving a constant current through the tissue followed by the reverse current to maintain charge balance.

Currently, most retinal prosthetic approaches can be categorized into two types based on the method of receiving visual information. The first type, which is adopted by most of the leading groups, uses an external camera for image acquisition and processing, after which electrical signals are sent to an implanted microelectrode array, which in turn stimulates ganglion cells..$^{(9-12)}$ The second type of retinal prosthesis uses photodiode arrays directly implanted at the retina of an eye, to provide direct ganglion cell stimulation. ${ }^{(13-15)}$ The second type is preferred over the first type, since the first type requires additional functionalities, such as image acquisition, data processing and data transmission. However, the research results to date show that the direct photodiode methods still have several limitations to overcome. Stelzle et al. ${ }^{(13)}$ and Wilke et al. ${ }^{(14)}$ have presented a retinal prosthetic system consisting of a direct-stimulation (DS) array and micro-photodiode array (MPDA) to investigate the ability to reliably recognize complex spatial percepts. However, the system requires an added on-chip analog amplifier to produce sufficiently large current to stimulate the ganglion cells. Khraiche et al., have developed a high-sensitivity photodetector using a vertical silicon nanowire array. ${ }^{(15)}$ However, they only acknowledged the photosensitivity, and did not deal with the details of photoresponsivity, which is the most important characteristic for a photodetector to be used in retinal prosthetics.

Our research group has very recently proposed the use of a novel nanowire as a photoreceptor, ${ }^{(16)}$ in which we fabricated a nanowire photodetector, measured its photosensitivity, and discussed the use of this nanowire photodetector as a stand-alone retinal stimulation system without an external camera. In this study, we develop this novel nanowire-based photodetector on a flexible substrate for retinal implant, and present performance evaluation results to show that the proposed device can be used in a retinal prosthesis. 


\section{Concept and Design}

The retina is an extension of the central nervous system in the eye. The retina converts light information to electrical signals that the optic nerve transports to the visual cortex of the brain, as shown in Fig. 1. ${ }^{(17)}$ In a healthy retina, the photoreceptors modulate the released neurotransmitters in response to light and stimulate the horizontal and bipolar cell layers, which in turn stimulate the ganglion cell layer that possesses axonal extensions leading all the way to the visual cortex of the brain. ${ }^{(18)}$ Thus, the loss of photoreceptors in the retina through diseases such as ARMD and RP renders the retina incapable of detecting visual information.

In this paper, a novel retinal prosthetic system with a silicon nanowire photodetector is proposed to replace the function of degenerated photoreceptor cells in the retina, i.e., modulation and transmission of visual signals. The proposed photodetector-based retinal prosthetic system consists of three elements: a silicon nanowire photodetector array, a 3D microelectrode array, ${ }^{(19)}$ and a power source, as illustrated in Fig. 2(a). In Fig. 2(b), the design concept of the $N \times N$ matrix structure and single pixel consisting of a silicon nanowire photodetector and microelectrode is shown to implement the proposed highdensity retinal prosthetic system. The silicon nanowire photodetector and microelectrode arrays are implanted in the retina of an eye, and the power source (i.e., battery and electronics) is attached on the eye near the implanted microsystem as the power supply and for signal processing.

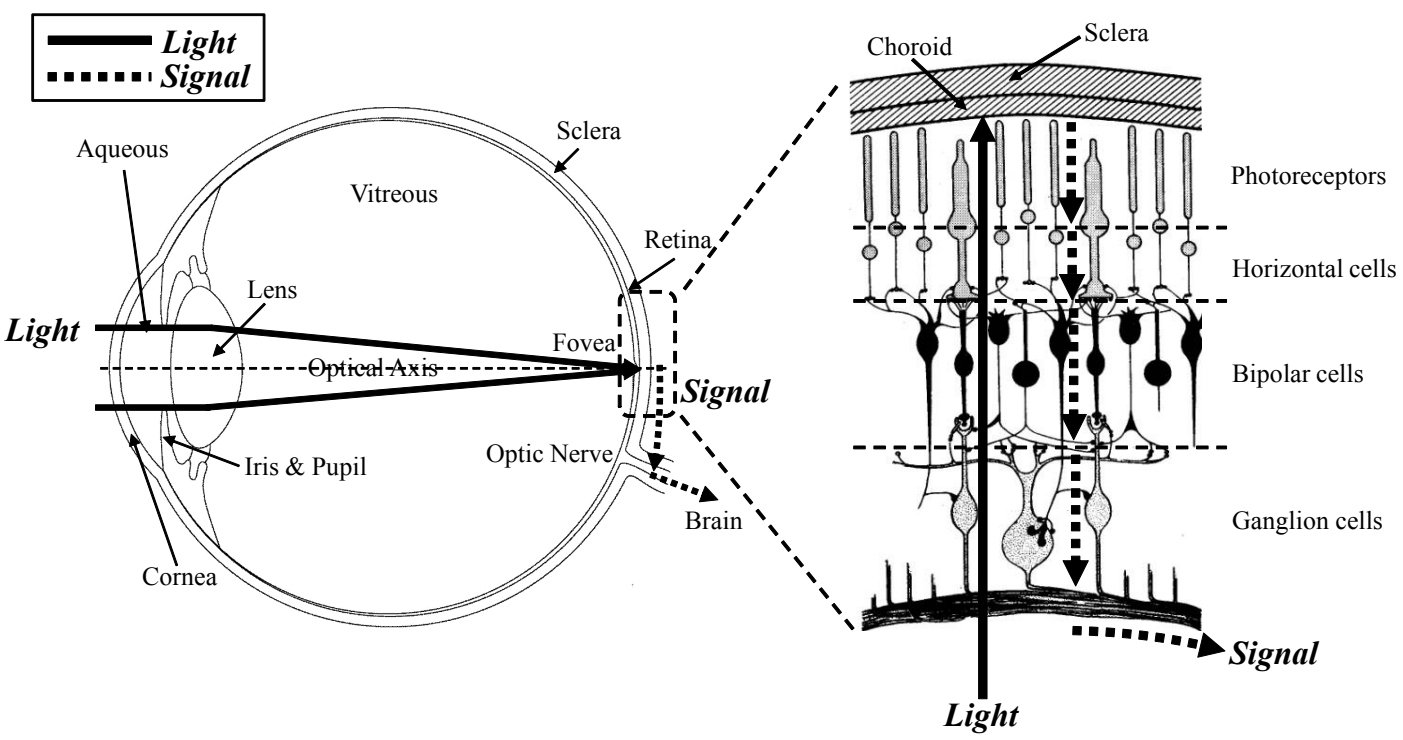

Fig. 1. Cross-sectional schematic of eye and neural layer of retina. ${ }^{(17)}$ 


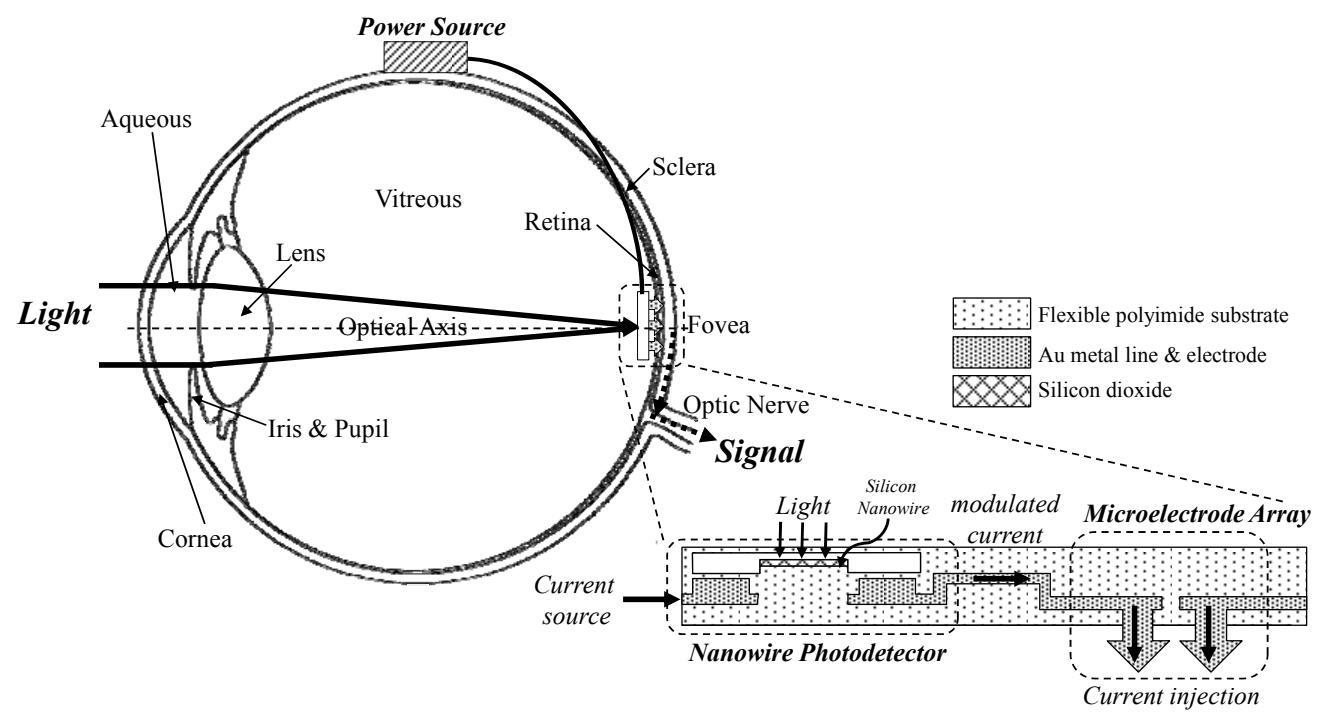

(a)

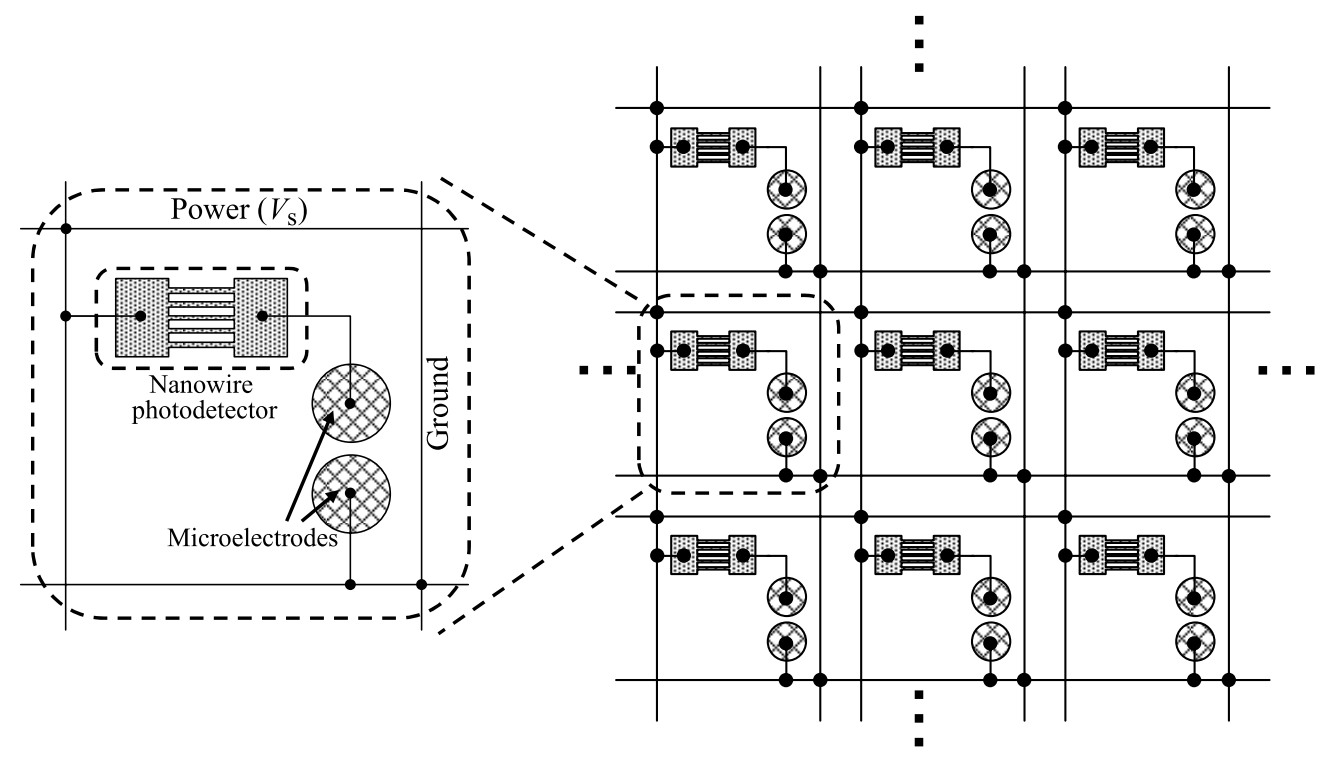

(b)

Fig. 2. Schematic diagrams of the proposed retinal prosthetic system. (a) Proposed photodetectorbased retinal prosthetic system. (b) Schematic of unit cell and $N \times N$ array. 
The detailed signal processing method of the photodetector-based retinal prosthetic system is shown in Fig. 3. A single pixel of the proposed system can be modeled as an equivalent circuit consisting of a variable resistor $\left(R_{\mathrm{NW}}\right)$ and characteristic impedance $\left(Z_{\mathrm{C}}\right)$, as illustrated in Fig. 3(a). The resistance of the variable resistor $\left(R_{\mathrm{NW}}\right)$ is adjusted

(a)

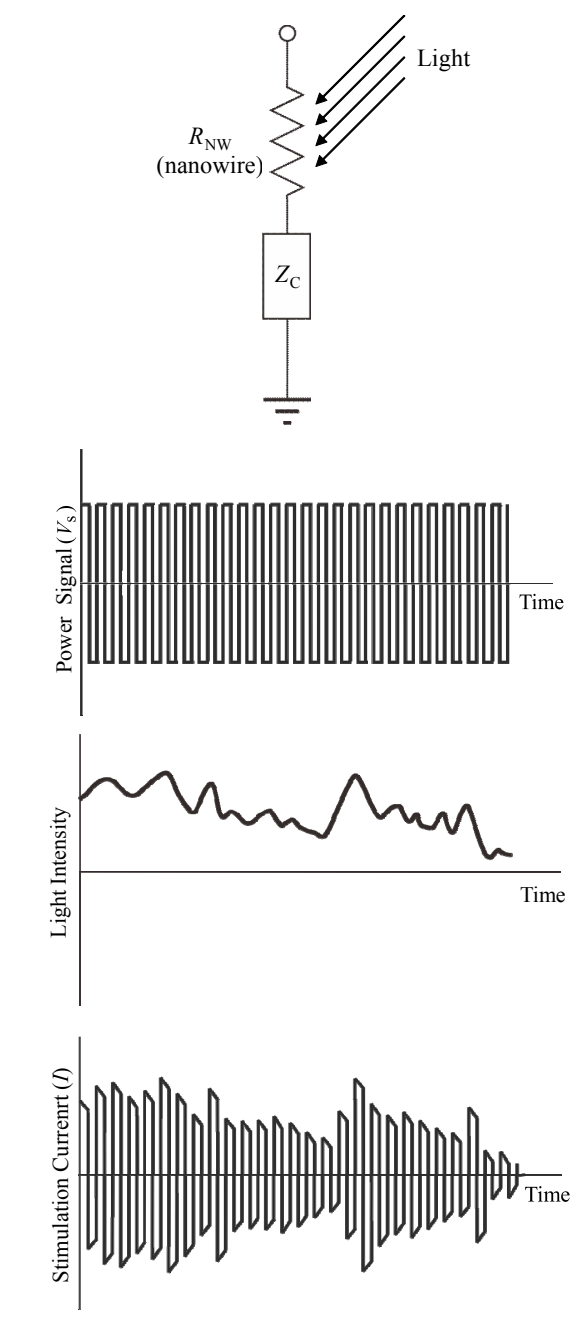

(b)

(c)

Fig. 3. Signal processing scheme of photodetector-based retinal prosthetic system. (a) Equivalent circuit model of nanowire photodetector and microelectrode. (b) Square pulse generated by power source. (c) Arbitrary intensity of light illuminated to the photodetector. (d) Modulated electrical signal by the photodetector. 
by changing the intensity of light. The silicon nanowire photodetector shows constant resistance when no outer light is illuminated. However, when outer light illuminates the nanowire array, the resistance of the nanowire array is decreased owing to electronhole pair generation, which, in turn, results in an increment of the conductivity of the nanowire array. The characteristic impedance $\left(Z_{\mathrm{C}}\right)$ is the total impedance comprising the intrinsic impedance of retinal tissue and the interface impedance between the implanted microelectrode array and retinal tissue.

The biphasic signal is generated by the power source shown Fig. 2(a). The biphasic signal $\left(V_{\mathrm{S}}\right)$ is a square pulse (or sinusoidal wave) that has a specific amplitude and frequency for efficient electrical stimulation of retinal cells (Fig. 3(b)). As an arbitrary intensity of light, as shown in Fig. 3(c), is illuminated onto a nanowire array, the resistance of the nanowire changes depending on the amplitude of light intensity, which results in conductivity variation in the nanowire photodetector. The square pulse generated by the power source is modulated by the conductivity variation of the nanowire photodetector, and the modulated electrical signal (Fig. 3(d)) is delivered to the microelectrode array for retinal stimulation. In brief, the electrical signal is generated by the power source, and the signal is modulated by the silicon nanowire photodetector depending on the illuminated light intensity for electrical stimulation of the neuronal network in the retina via the microelectrode array.

\section{Fabrication}

To be suitable for implantation into the eye and to fit the curvature of the eyeball, it is necessary to implement a retinal prosthetic system with a silicon nanowire photodetector on a flexible substrate. The top schematic view of the nanowire photodetector is shown in Fig. 4. For the fabrication of a silicon nanowire photodetector on a flexible substrate, silicon nanowire arrays are fabricated on a rigid silicon substrate first, by a top-down process. Then the silicon nanowire photodetector is transferred onto a flexible substrate by polyimide patterning and substrate removal. The detailed fabrication process is shown in Fig. 5.

First, a p-type silicon nanowire array is fabricated by the top-down process involving photolithography, silicon dry etching, anisotropic wet etching, and thermal oxidation [20]. A boron-doped (100)-oriented silicon substrate is prepared, a 1000- $\AA$-thick thermal oxide is grown by a wet oxidation process (Fig. 5(a)), and $1.5 \mu \mathrm{m}$ line patterns are defined by photolithography (Fig. 5(b)). Rectangular silicon columns $(1.5 \mu \mathrm{m}$ in width and $2.1 \mu \mathrm{m}$ in depth) are fabricated by silicon deep reactive ion etching (DRIE) (Fig. 5(c)). Subsequently, anisotropic silicon wet etching using tetra-methyl-ammoniumhydroxide (TMAH) solution is performed for $90 \mathrm{~s}$ (Fig. 5(d)). The rectangular shape of silicon structures is etched by exploiting the anisotropic wet etching properties of silicon in a TMAH solution. Anisotropic silicon wet etching is performed until the (111) planes, which have the slowest etch rate in TMAH solution, are revealed at the center of the structure. After the fabrication of hourglass-shaped silicon structures, the silicon nanowire array is fabricated by wet oxidation (Fig. 5(e)). In this process, the entire silicon surface is thermally oxidized with a 2000 - $\AA$-thick silicon dioxide layer and 
triangular silicon nanowires are fabricated. A fabricated silicon nanowire is shown in Fig. 6(a). Its size is approximately $150 \mathrm{~nm}$ in width, which can be precisely controlled by the oxidation time. After the fabrication of the silicon nanowire array, the silicon substrate is bonded with another silicon wafer with a 3000-A-thick thermal oxide layer (Fig. $5(\mathrm{f})$ ). Then, the bonded wafer is thinned by lapping and chemical mechanical polishing (CMP) to remove the current path through the silicon substrate (Fig. 5(g)). After the silicon area covering the silicon nanowire array is etched by silicon DRIE (Fig. 5(h)), the 3000- $\AA$-thick aluminum (Al) metal electrodes are patterned on both sides of the broad silicon area (Fig. 5(i)). After the $\mathrm{Al}$ metal electrodes are patterned, rapid thermal annealing (RTA) is performed at $400^{\circ} \mathrm{C}$ for $5 \mathrm{~min}$ in $\mathrm{N}_{2}$ condition to lower the contact resistance between the electrode and silicon substrate by diffusing $\mathrm{Al}$ metal into silicon. In Figs. 6(b) and 6(c), the fabricated silicon nanowire photodetector on a rigid silicon substrate is shown. The length of the nanowire is $20 \mu \mathrm{m}$ and 250 nanowires are equally spaced with intervals of $3.2 \mu \mathrm{m}$.

To transfer the fabricated silicon nanowire array from a rigid substrate to a flexible substrate, polyimide is coated and patterned on the bonded wafer (Fig. 5(j)). After the polyimide patterning process, the polyimide layer is cured at $350^{\circ} \mathrm{C}$ for $2 \mathrm{~h}$ in a vacuum

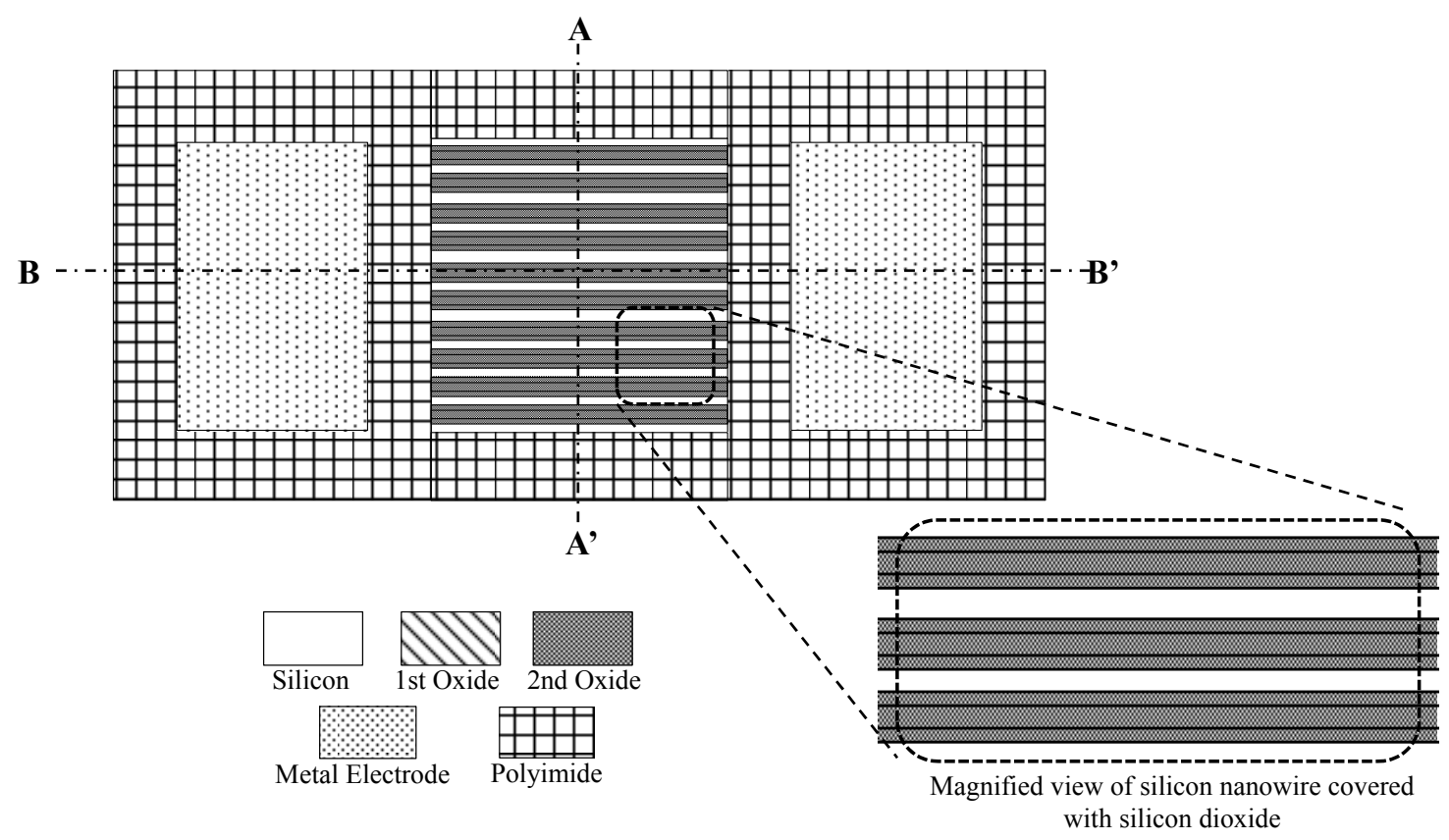

Fig. 4. Top schematic view of silicon nanowire photodetector on a flexible substrate. 
I. Nanowire array fabrication (Cross-sectional view along AA' in Fig. 4)

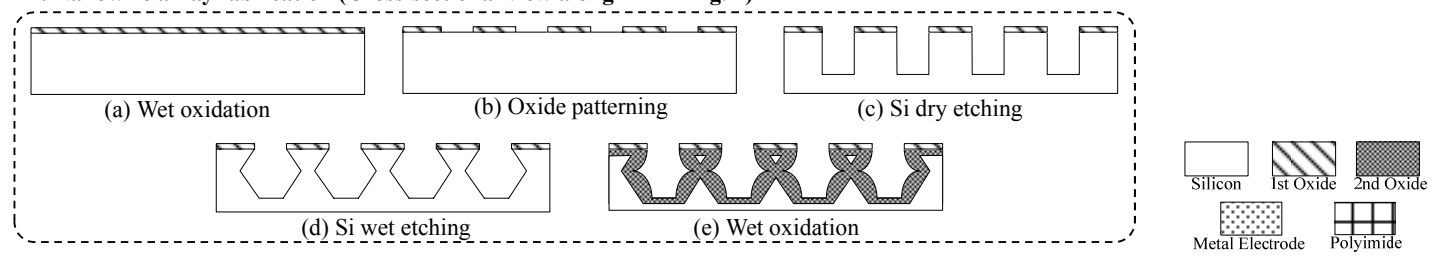

II. Substrate transfer process

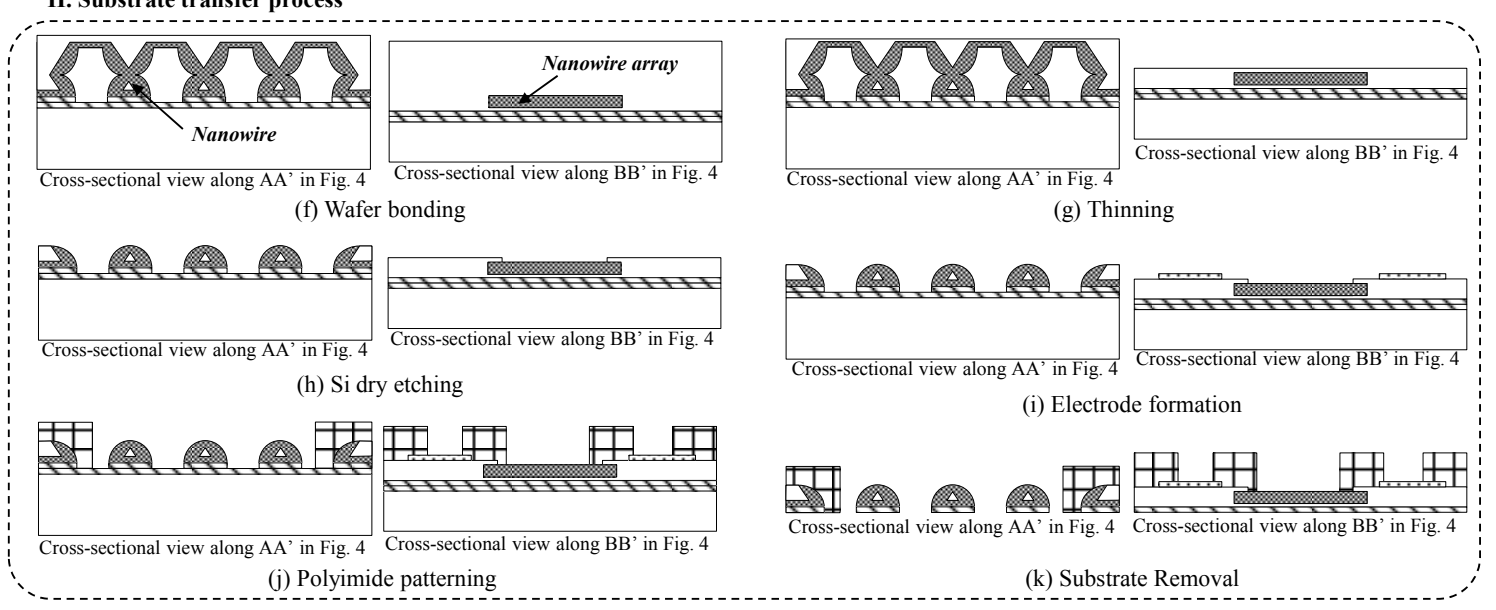

Fig. 5. Fabrication process of silicon nanowire photodetectors on a flexible substrate.

oven to give it mechanical and chemical durability. During the curing process, the solvent component in the polyimide is vaporized by thermal heating, which results in a diminished polyimide layer thickness. Finally, after the bottom silicon substrate is removed by silicon DRIE (Fig. 5(k)), the silicon nanowire photodetector on a flexible substrate is obtained, as shown in Fig. 6(d). The flexible silicon nanowire photodetector is bent by the residual stress between the polyimide film and the remaining silicon layer, with a bending radius of about $5 \mathrm{~mm}$.

\section{Performance Evaluation}

The photoreceptors in the retina operate over an extremely wide range of illumination with high visual acuity.(1) To evaluate the feasibility of the flexible silicon nanowire photodetector for implementation of a retinal prosthetic system, the optical and mechanical characteristics are measured.

The optical characteristics of the silicon nanowire photodetector on a rigid substrate are measured first before transferring it onto a flexible substrate. The silicon nanowire 


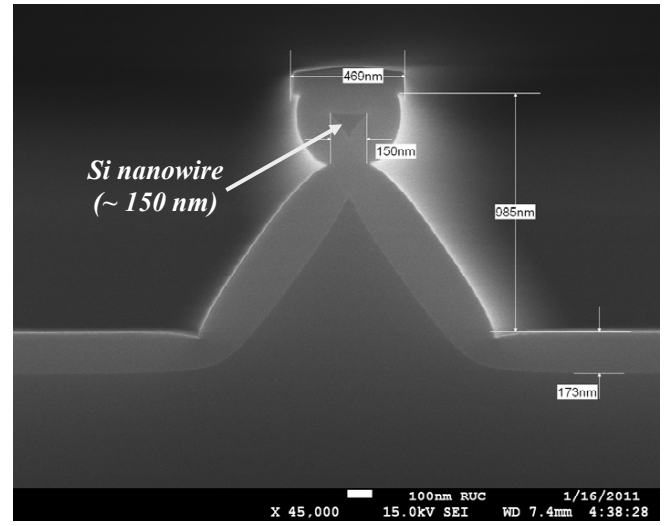

(a)

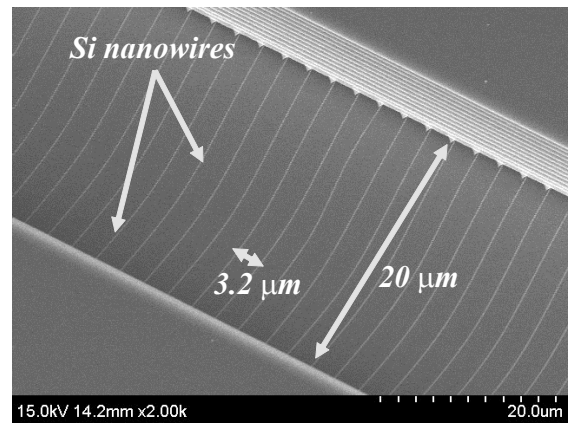

(c)

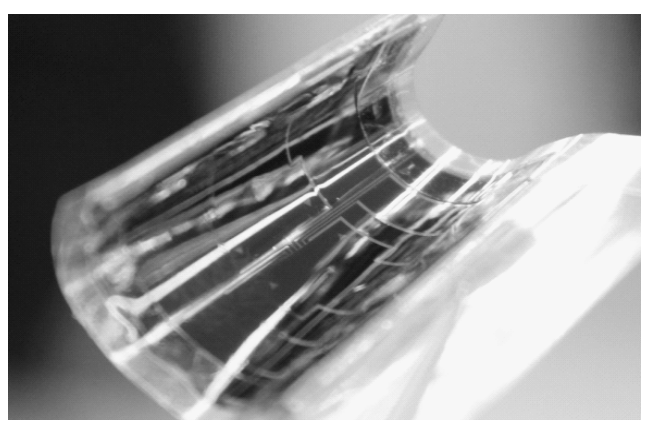

(d)

Fig. 6. Fabrication results. (a) Fabricated silicon nanowire. (b) Nanowire photodetector fabricated on rigid substrate. (c) SEM image of fabricated nanowire photodetector. (d) Fabricated nanowire photodetector on flexible substrate.

photodetector is illuminated by a white light (halogen light) source with a range of light intensity between 0 and $1.6 \mathrm{~mW} / \mathrm{cm}^{2}$ and a bias voltage of $1 \mathrm{~V}$. In Fig. 7(a), the IV characteristic of the silicon nanowire photodetector for various intensities of light is shown. Each intensity of white light is measured before the experiment using an illuminometer for precise examination of the IV characteristic of the fabricated device. As the intensity of light is increased, the resistance of the silicon nanowire is decreased owing to electron-hole pair generation, which results in an increment of the current passing through the silicon nanowire. In Fig. 7(b), the photocurrent response depending on the light intensity of the fabricated device is shown. The result of the modulated current output is not sufficient for stimulating the ganglion cells in a retina. To properly stimulate the ganglion cells, the system requires an integrated amplifying circuitry. 


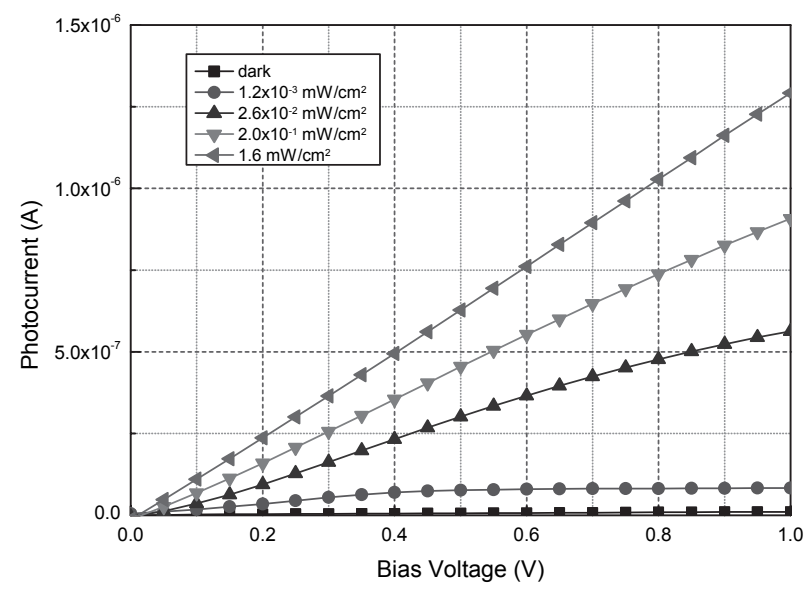

(a)
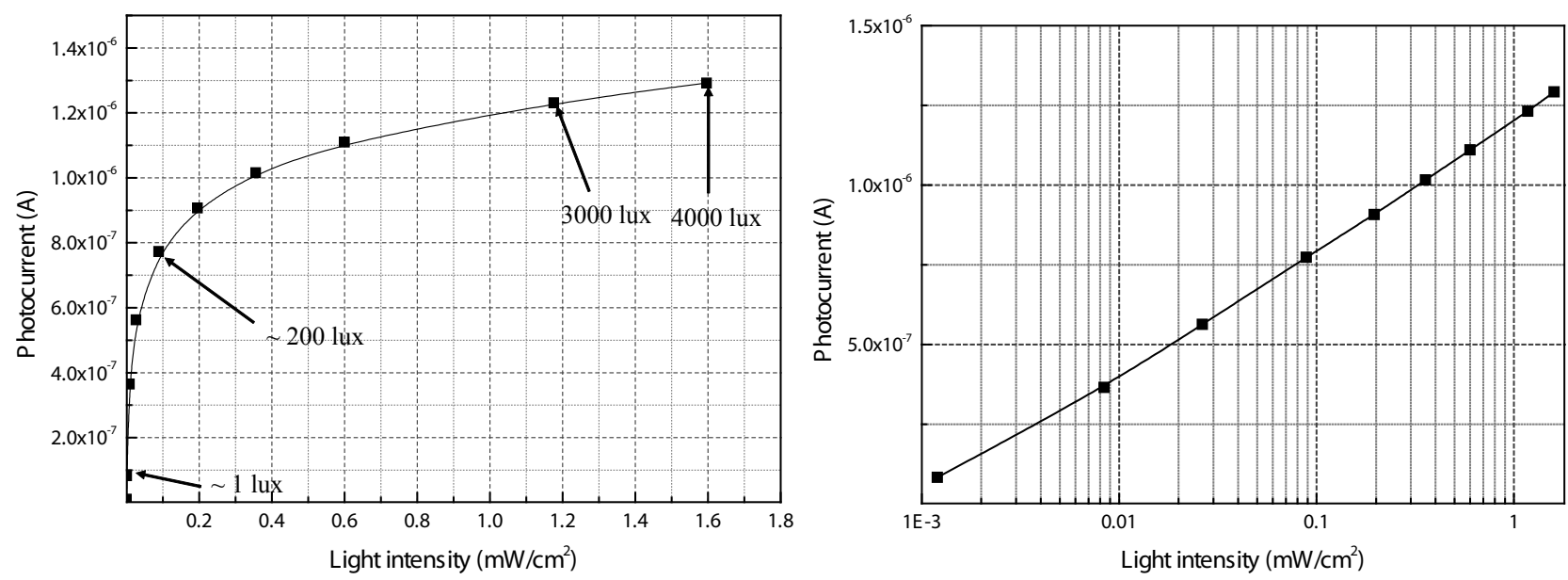

(b)

Fig. 7. Optical characteristics of the nanowire photodetector on a rigid substrate. [Bias voltage, $1 \mathrm{~V}$, normal scale on $x$-axis (upper), log scale on $x$-axis to magnify the low light intensity region (lower)]. (a) IV characteristics. (b) Photosensitivity.

However, the measured photocurrent under such a low light intensity of $1.2 \mu \mathrm{W} / \mathrm{cm}^{2}$ is clearly distinguishable, and the photocurrent under the light intensity of $1.6 \mathrm{~mW} / \mathrm{cm}^{2}$ and a bias voltage of $1 \mathrm{~V}$ is increased by about one thousand times compared with that under the dark condition $\left(0 \mathrm{~mW} / \mathrm{cm}^{2}\right)$. The turn-off current level under the dark condition is measured to be about $10^{-8} \mathrm{~A}$, which is below the threshold to stimulate or harm the 
ganglion cell in the retina. ${ }^{(21)}$ The silicon nanowire photodetector is highly photosensitive under low light intensity, which might be beneficial at low light conditions. Although the results do not show a perfect linearity, the photocurrent is uniquely valued and monotonically increased for the entire measured intensity spectrum, which indicates that the fabricated nanowire photodetector can operate under an extremely wide range of light intensity.

For a precise measurement of the photosensitivity and photoresponsivity of the silicon nanowire photodetector, the fabricated device is illuminated using a monochromator. To evaluate the IV characteristics, light with wavelengths of $400,500,600$, and 800 $\mathrm{nm}$ is illuminated with a monochromator, and their corresponding photocurrents are measured. As shown in Fig. 8(a), a clearly distinguishable photocurrent is measured for each wavelength, although the intensity of illuminated light is very small (10-30 $\mathrm{nW} / \mathrm{cm}^{2}$ ). In Fig. 8(b), the photoresponsivity of the nanowire photodetector to a specific wavelength is shown. The average photoresponsivity is over $1 \times 10^{4} \mathrm{~A} / \mathrm{W}$ in all measured wavelength ranges, and in particular, for a wavelength of $400 \mathrm{~nm}$, the photoresponsivity is over $1 \times 10^{5} \mathrm{~A} / \mathrm{W}$. The fabricated silicon nanowire photodetector offers superior photoresponsivity compared with a PIN photodiode and an avalanche photodiode (APD), which show maximum photoresponsivities of $1 \mathrm{~A} / \mathrm{W}$ and $10 \mathrm{~A} / \mathrm{W}$ at a wavelength between 700 and $800 \mathrm{~nm}$, respectively. ${ }^{(22,23)}$ However, the silicon nanowire photodetector shows its maximum photosensitivity and photoresponsivity in a low-wavelength range. It is assumed that since the width of the nanowire is small (approximately $150 \mathrm{~nm}$ ), more light with a lower wavelength is absorbed into the silicon nanowire leading to higher photosensitivity and photoresponsivity.

To examine the preserved optical characteristics after transferring the nanowire photodetector from a rigid substrate to a flexible substrate, the photosensitivity is measured before and after the substrate transfer (Fig. 9). The electrical evaluation is performed by IV-characteristic measurement under the dark condition $\left(0 \mathrm{~mW} / \mathrm{cm}^{2}\right)$, and optical evaluation is performed by photocurrent measurement under a light intensity between 0 and $1.6 \mathrm{~mW} / \mathrm{cm}^{2}$ and a bias voltage of $1 \mathrm{~V}$. Each experiment is carried out with three devices, as shown in Fig. 8. Device 1 shows a decreased current level for both electrical and optical evaluation during substrate transfer (Fig. 9(a)). Device 2 shows an increased current level for electrical evaluation but a similar current level for optical evaluation (Fig. 9(b)). Device 3 show an increased current level for electrical evaluation but a decreased current level for optical evaluation (Fig. 9(c)). The increment or decrement of the current level before and after the substrate transfer of the silicon nanowire seems to be due to the high-temperature annealing process during polyimide curing and the bending stress from the residual stress of polyimide after transfer to a flexible form. However, despite the change in the current level after transferring the silicon nanowire photodetector to a flexible substrate, the results indicate that the performance characteristics of the silicon nanowire photodetector are well preserved.

To verify the mechanical durability of the nanowire photodetector on a flexible substrate, the mechanical bending tests are carried out 200 times. The experiment is performed under a light intensity of $1 \mathrm{~mW} / \mathrm{cm}^{2}$ and bias voltage of $1 \mathrm{~V}$, and the photocurrent response during the repeated bending is measured. The bending tests 


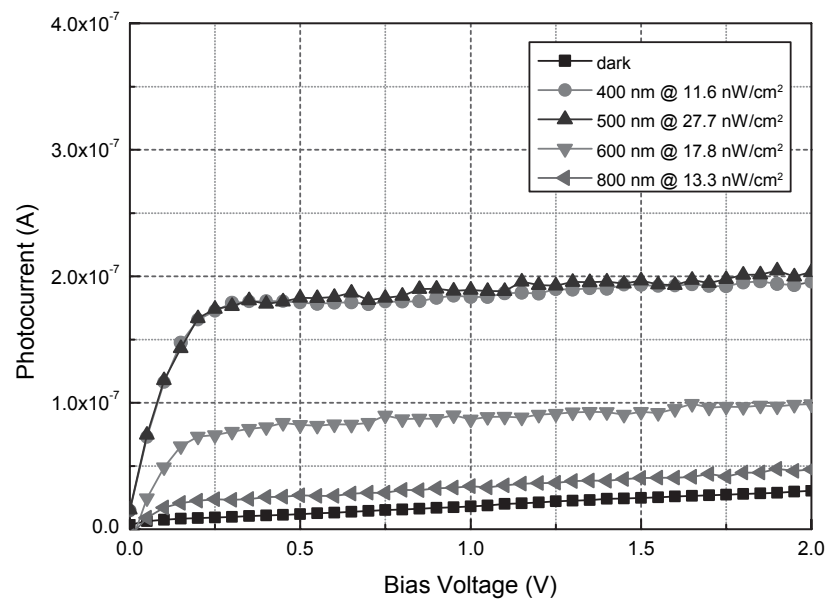

(a)

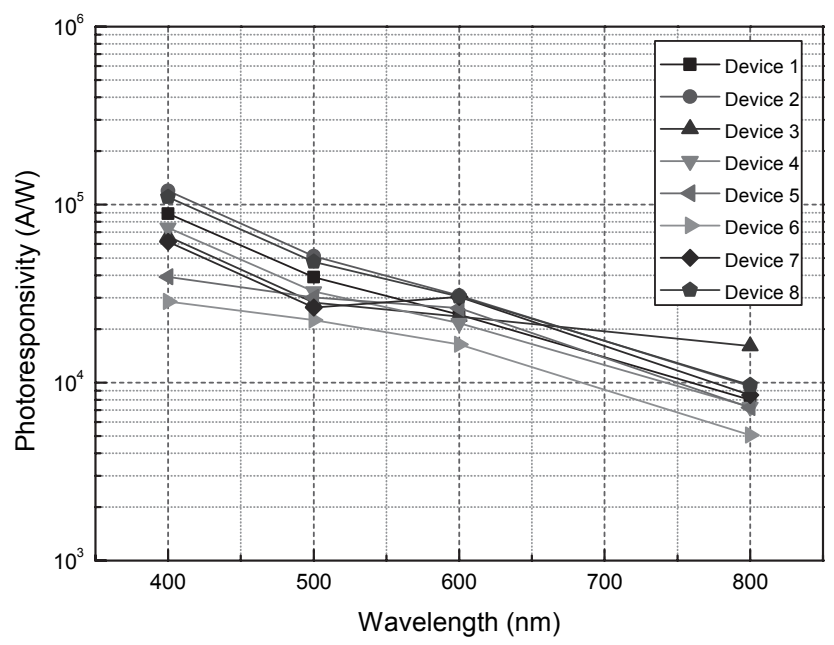

(b)

Fig. 8. Optical characteristics of the nanowire photodetector using monochromator. (a) IV characteristics. (b) Photoresponsivity (Bias voltage, $2 \mathrm{~V}$ ).

are performed by repeatedly moving a probe pin, which holds each end of the flexible photodetector device, as shown in lower three images of Fig. 10. The result reveals that the optical characteristic is well preserved even after a number of bending tests, as shown in Fig. 10. 

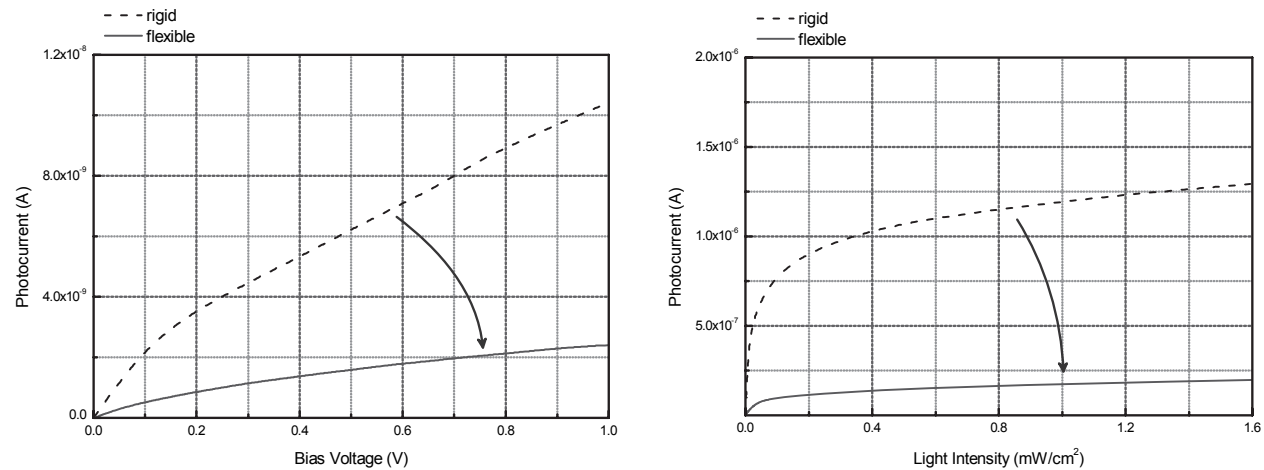

(a)
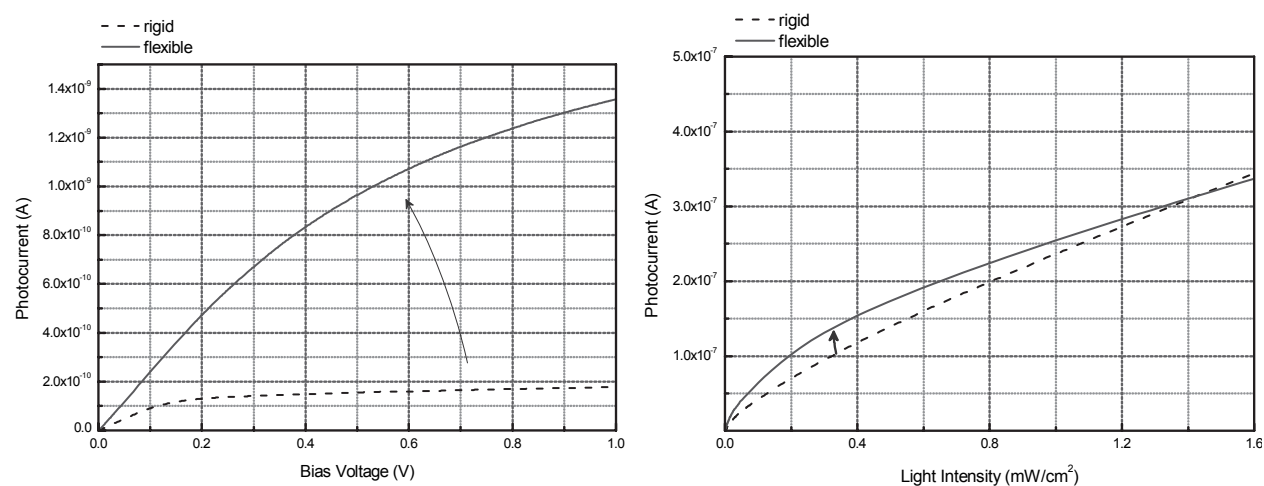

(b)
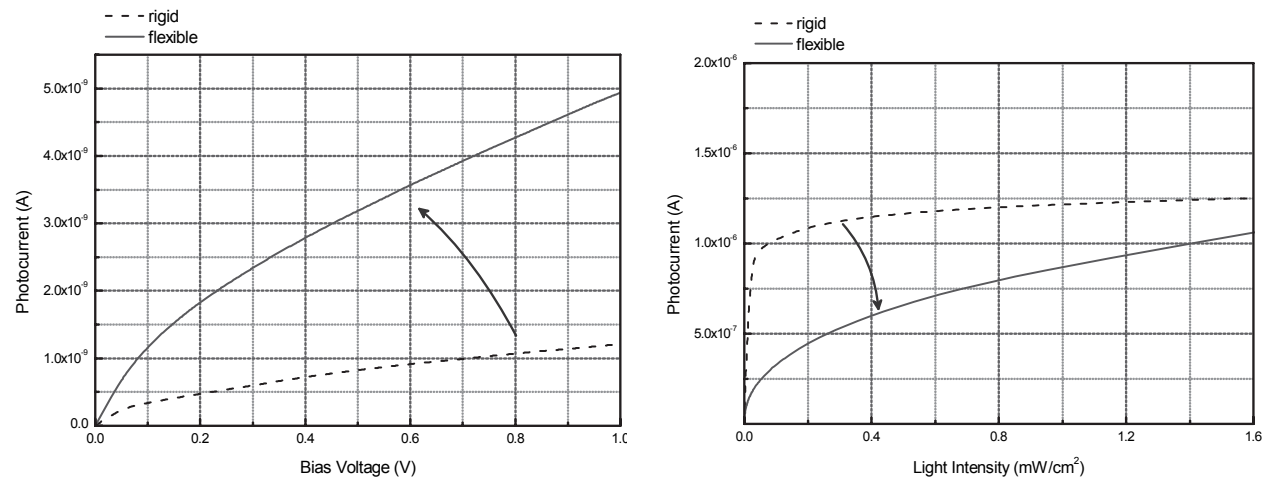

(c)

Fig. 9. Performance characteristics of the nanowire photodetector before and after transfer from a rigid substrate to a flexible substrate. (a) IV characteristic (left) and photosensitivity (right) of device 1. (b) IV characteristic (left) and photosensitivity (right) of device 2. (c) IV characteristic (left) and photosensitivity (right) of device 3 . 


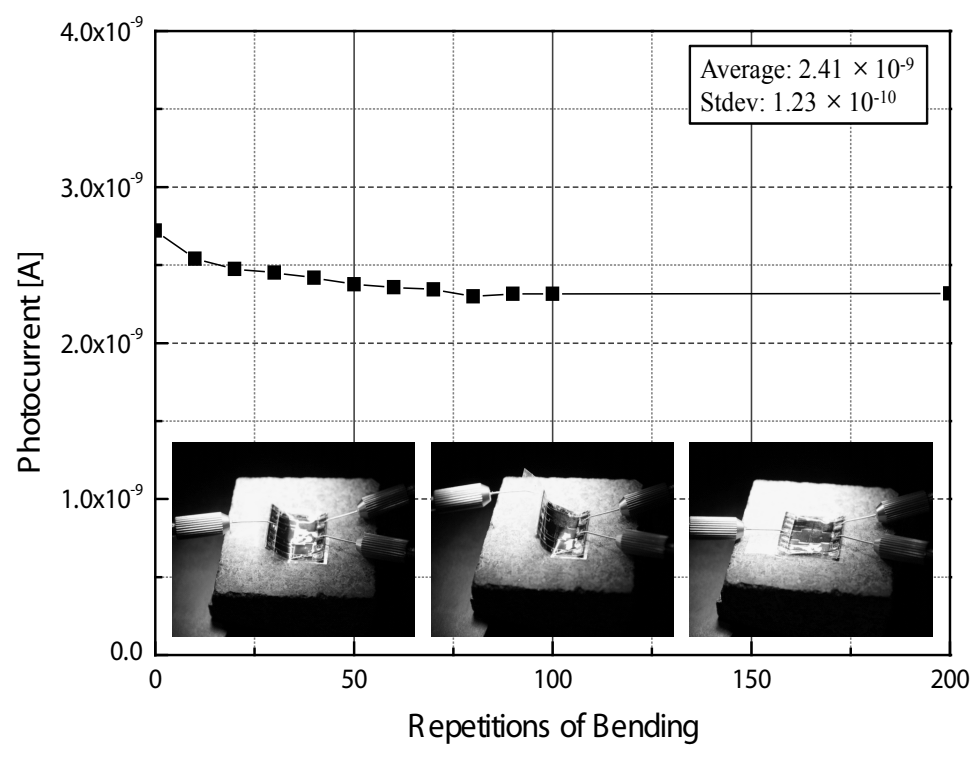

Fig. 10. Bending test results of the flexible nanowire photodetector (Lower three images show a single bending test procedure).

\section{Conclusions}

In this paper, a silicon nanowire photodetector on a flexible substrate for a retinal prosthetic system was presented. We developed a novel nanowire photodetector on a flexible substrate for retinal implant, and presented performance evaluation results to show that the proposed photodetectors can indeed be used in retinal prostheses. To fabricate silicon nanowire photodetectors on a flexible substrate, silicon nanowire photodetectors are first fabricated on a rigid silicon substrate by a top-down process. Then, the silicon nanowire photodetectors were transferred from a rigid substrate to a flexible substrate by polyimide patterning and substrate removal.

To evaluate the feasibility of using the silicon nanowire photodetector in a retinal prosthesis, first the electrical and optical characteristics were measured. The device showed high photosensitivity and photoresponsivity over a wide range of light intensity. In particular, photocurrent was clearly distinguishable even under a very low light intensity of $10-30 \mathrm{nW} / \mathrm{cm}^{2}$, and the average photoresponsivity was measured to be approximately $1 \times 10^{4} \mathrm{~A} / \mathrm{W}$. To examine the preserved optical characteristics after transferring the nanowire photodetector from a rigid substrate to a flexible substrate, the IV characteristic and photosensitivity were measured. The results indicated that 
the electrical and optical characteristics are well maintained after the transfer. In order to verify the mechanical durability of the silicon nanowire photodetector on a flexible substrate, mechanical bending tests were performed. The optical characteristic of the flexible photodetector was well preserved during the 200 bending tests. The presented performance evaluation results revealed that the proposed novel nanowirebased photodetector is suitable for implementation in the retinal prosthetic system. The proposed novel method can result in the realization of a stand-alone, miniature retinal prosthetic system without the need to use an external camera, paving the way to a revolutionary breakthrough in the development of the artificial retina.

\section{Acknowledgements}

This research was supported by the Happy Tech. program through the National Research Foundation of Korea (NRF) funded by the Ministry of Education, Science and Technology (2010-0020852). This work was also supported by the R\&D program of the Korea Ministry of Knowledge and Economy (MKE) and the Korea Evaluation Institute of Industrial Technology (KEIT) (Grant No. 10035501).

\section{References}

1 M. M. LaVail, J. G. Hollyeld and R. E. Anderson: Retinal Degeneration: Experimental and Clinical Studies (John Wiley \& Sons Inc., USA, 1985) Chap. 1.

2 J. Winter, S. Cogan and J. Rizzo: Journal of Biomaterials Science, Polymer Edition 18 (2007) 1031.

3 A. H. C. Koh and C. L. Ang: Ann. Acad. Med. Singapore 31 (2002) 399.

4 J. L. Stone, W. E. Barlow, M. S. Humayun, E. de Juan, Jr. and A. H. Milam: Arch. Ophthalmol. 110 (1992) 1634.

5 A. Santos, M. S. Humayun, E. de Juan, Jr., R. J. Greenburg, M. J. Marsh, I. B. Klock and A. H. Milam: Arch. Ophthalmol. 115 (1997) 511.

6 S. Y. Kim, S. Sadda, J. Pearlman, M. S. Humayun, E. de Juan, Jr., B. M. Melia and W. R. Green: Retina 22 (2002) 471.

7 E. Zrenner, A. Stett, S. Weiss, R. B. Aramant, E. Guenther, K. Kohler, K. D. Miliczek, M. J. Seiler and H. Haemmerle: Vis. Res. 39 (1999) 2555.

8 M. Humayun, R. Propst, E. de Juan, Jr., K. McCormick and D. Hickingbotham: Arch. Ophthalmol. 112 (1994) 110.

9 J. F. Rizzo III, J. Wyatt, J. Loewenstein, S. Kelly and D. Shire: Invest. Ophthalmol. Vis. Sci. 44 (2003) 5355.

10 M. S. Humayun, J. D. Weiland, G. Y. Fujii, R. Greenberg, R. Williamson, J. Little, B. Mech, V. Cimmarusti, G. Van Boemel, G. Dagnelie and E. de Juan: Vision Res. 43 (2003) 2573.

11 J. M. Seo, J. Zhou, E. T. Kim, K. I. Koo, J. H. Ye, S. J. Kim, H. Chung, D. Cho, Y. S. Goo and Y. S. Yu: Visual Prosthesis and Opthalmic Devices, New Hope in Sight (Humana Press Inc., New Jersey, 2007) Chap. 8.

12 G. J. Suaning, N. H. Lovell, K. Schindhelm and M. T. Coroneo: Aust. New Zealand J. Ophthalmol. 26 (1998) 195.

13 M. Stelzle, A. Stett, B. Brunner, M. Graf and W. Nisch: Biomedical Microdevices 3 (2001) 133. 
14 R. Wilke, V.-P. Gabel, H. Sachs, K.-U. B. Schmidt, F. Gekeler, D. Besch, P. Szurman, A. Stett, B. Wilhelm, T. Peters, A. Harscher, U. Greppmaier, S. Kibbel, H. Benav, A. Bruckmann, K. Stingl, A. Kusnyerik and E. Zrenner: Invest. Ophthalmol. Vis. Sci. 52 (2011) 5995.

15 M. L, Khraiche, Y. Lo, D. Wang, G. Cauwenberghs, W. Freeman and G. A. Silva: 33rd Annual International Conference of the IEEE EMBS 2011 (IEEE EMBS, Boston, USA, 2011) p. 2933.

16 S. W. Jung, K. I. Koo, S. K. Park, S. Lee, J. H. Ahn, S. J. Hong, H. J. Yoo, M. H. Lee and D. Cho: The 13th Korean MEMS Conference (KMEMS, Jeju, Korea, 2011) p. 181.

17 J.-U. Meyer: Sens. Actuators, A 97-98 (2002) 1.

18 J. C. Martins and L. A. Sousa: Bioelectronics Vision - Retina Models, Evaluation Metrics, and System Design (World Scientific Publishing Co. Pte. Ltd., Singapore, 2009) Chap. 2.

19 K. Koo, S. Lee, S. H. Bae, J. M. Seo, H. Cung and D. Cho: JMEMS 20 (2011) 251.

20 K. N. Lee, S. W. Jung, K. S. Shin, W. H. Kim, M. H. Lee and W. K. Seong: Small 4 (2008) 642.

21 R. J. Jensen, O. R. Ziv and J. F. Rizzo III: Invest. Ophthalomol. Vis. Sci. 46 (2005) 1486.

22 G. Wanlin, L. Giraudet, J. P. Praseuth, A. Miras, E. Legros: 11th International Conference on Integrated Optics and Optical Fibre Communications and 23rd European Conference on Optical Communications (IOOC-ECOC 97, Edinburgh, UK, 1997) p. 37.

23 J. Wei, F. Xia and S. R. Forrest: IEEE Phontonics Technol. Lett. 14 (2002) 1590. 